\title{
Influence of camera rotation on stereo-DIC and compensation methods
}

\author{
R. Balcaen • P. L. Reu • P. Lava • D. Debruyne
}

Received: date / Accepted: date

\begin{abstract}
Camera rotation during stereo digital image correlation (DIC) measurements is always present in some amount and is caused by environmental vibrations during testing (e.g. blast testing, testing in industrial environments, vibration testing [1], etc.). The impact of changing extrinsic parameters on stereo-DIC measurements was investigated in this paper by simulating several DIC experiments with a shaking camera system. To obtain an appropriate order of magnitude of the camera motion, the actual camera rotation during a test was observed and reported. The impact hereof was investigated by analyzing static images and by simulating camera rotation with a stereo-DIC simulator presented in [2]. Insight on this underestimated error source is explained by investigating which rotations are more critical to the resulting displacements and strains. Two possible compensation methods were investigated and their capability of compensating camera motion was evaluated.
\end{abstract}

Keywords Digital image correlation · Uncertainty quantification · Camera rotation · Compensation of shaky cameras · Error assessment

R. Balcaen · P.Lava · D.Debruyne

KU Leuven, Department of Materials Technology, Campus Ghent, Gebroeders Desmetstraat 1, 9000 Ghent, Belgium

E-mail: ruben.balcaen@kuleuven.be

P. Lava

MatchID, Wijmenstraat 21T, 9030, Gent, Belgium

P. L Reu

Sandia National Laboratories, Albuquerque, New Mexico, United States 


\section{Introduction}

Stereo digital image correlation (stereo-DIC) is an optical-numerical technique to track a pattern applied to a surface to measure full-field displacement, deformation and strains [3]. Despite the acceptance and wide-spread use of the technique as a metrological tool [4] there are still some error sources that are underestimated and not yet fully understood. While most of the uncertainty quantification studies performed on stereo-DIC during the last few years focus on algorithm errors (e.g. $[2,5-7]$ ), we tend to focus on the experimental error sources since these are usually dominant in an experiment according to our experience. In this paper we investigated the influence of changing camera orientations, caused by environmental vibrations, on stereo-DIC results. Stereo-DIC includes a calibration- and triangulation-stage that complicates investigation of the numerical measurement chain, making it extremely hard to mathematically derive the influence of changing camera extrinsics. We therefore employ a stereo-DIC image generator, which is capable of imposing camera motion during a test (which could be caused by vibrations due to heavy machinery, cooling fans, camera heating [8] or other extreme environments [9]). The effect of out-of-plane motion in 2D-DIC was already investigated by Sutton et. al in [10], however there it is assumed that the extrinsic parameters remain constant during a test, while here we investigate the effect of relative camera motion in stereo-DIC, rather than 2D-DIC. The influence of camera rotation cannot be neglected, as previously noted by [5] as: "Structural vibrations ... dwarfed all other effects.". In this paper we address this problem, starting with measuring the actual camera rotation during experimental conditions in section 2 . Section 3 introduces the reader to the numerically generated benchmark images, which incorporate the measured camera rotation, to provide an understanding of the response of the stereo-DIC measurement system to camera rotation. Section 4 introduces two possible methods for reducing the effect of camera rotation on stereo-DIC results with higher accuracy by either numerically rectifying the images or by temporally averaging them. Please note that the focus of this paper is on describing the influence of camera rotation on the obtained displacements and strains with simulations of a representative experiment, rather than on all possible situations, because every setup behaves differently to environmental vibrations. 


\section{Presence of camera rotation during DIC experiments}

\subsection{Experimental setup}

Camera motion (both camera translation and camera rotation) will be present to some extent in all experiments; only high-tech laboratory facilities can achieve an almost vibration-free environment, which is rarely the case for more practical applications such as tensile tests, blast experiments, etc. To demonstrate the deleterious influence of camera rotation on (stereo-) DIC, a series of experimental studies were performed. An industrial test environment is mimicked by setting up a standard 2D-DIC rig on the ground, looking at a target (a speckled flat plate) standing on a floating optical table. The optical table served two purposes; it held two laser Doppler vibrometers (LDV) for measuring the movement of the camera body, while also restraining the target from moving. Vibrations were generated by a compressor standing on the ground a couple of feet away from the DIC rig. Images of the target were taken at 5FPS at the same time the LDVs measured the camera translation and rotation. This was done for three lens types; $16 \mathrm{~mm}$, $35 \mathrm{~mm}$ and $75 \mathrm{~mm}$. The setup can be seen in Figure 1, with the camera mounted on a tripod that stands on the ground, the target standing on the optical table and the LDVs spots located on the camera body for measuring the rotation around the optical axis. Six markers were attached to the camera in order to measure the rotation around the three principle axis. The green markers in Figure 2 are used for the rotation around the Z-axis, while the blue and red markers are used for identifying the rotation around the $\mathrm{Y}$ - and $\mathrm{X}$-axis respectively.

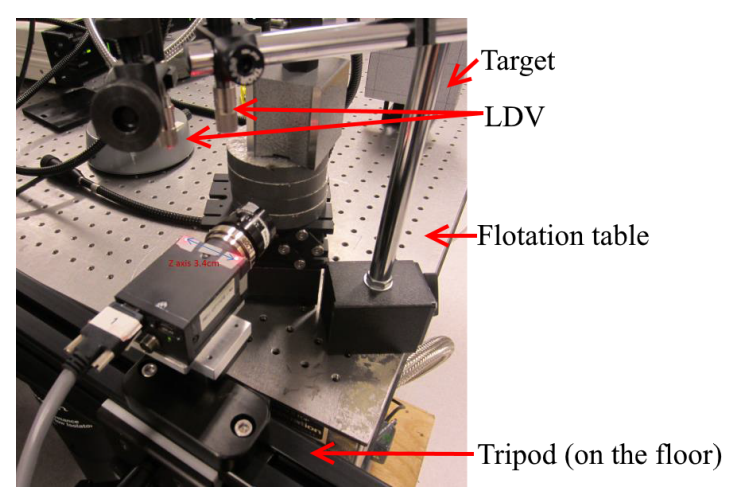

Fig. 1: Experimental setup industrial environment 


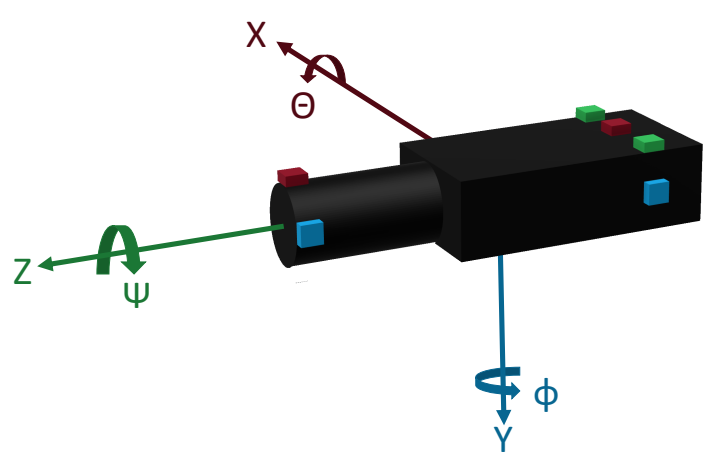

Fig. 2: Camera coordinate system

Polytec OFV-511 fiber interferometers were used in combination with OFV5000 controllers for measuring the velocity of the camera points. The data acquisition sample rate was $12.5 \mathrm{kHz}$ and the LDV controllers were set to fast tracking with low pass filtering and a sensitivity of $5 \mathrm{~mm} / \mathrm{s} / \mathrm{V}$. The relative displacement between those markers was then obtained by integrating the velocity. The rotation angle is consequentially calculated from the relative displacement as the arctangent of the fraction of the relative displacement and the distance between the LDVs on the camera (assuming the lasers were placed approximately around the center of rotation). The frequency response of the camera body in the Y-direction is displayed in Figure 3. However, note that the focus of this paper is on the influence of typical camera rotation and we are assuming that there is no resonance between the camera system and the compressor. Please also note that all rotations were measured in separate runs thus no synchronous data between different axes is available, although the input vibration was the same.

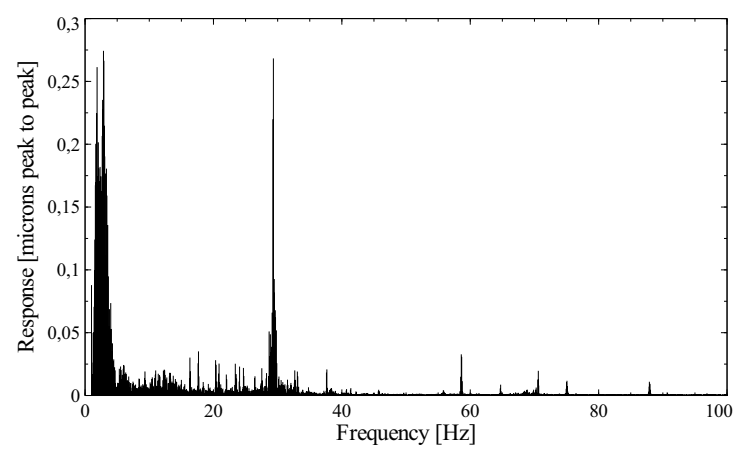

Fig. 3: Frequency response of the camera body in Y-direction 


\subsection{Results}

The measured rotations for the three different lenses can be seen in Figures 4(a) through 4(c), with a static test (in green squares) indicating the baseline data when there are no environmental vibrations. It is clear from Figure 4(a) that there is a sinusoidal rotation of the camera body around the horizontal in-plane axis (denoted as the rotation around the $\mathrm{X}$-axis as shown in Figure 2), tipping the camera lens up and down. It is also clear that this is the dominant rotation when comparing the magnitude of each rotation. The rotation around the vertical axis (denoted as rotation around the Y-axis) is less sinusoidal as shown in Figure 4(b), while also being lower in amplitude (about half of the amplitude of the rotation around the $\mathrm{X}$-axis). The rotation around the optical axis (denoted as rotation around the Z-axis) is composed of a lower frequency component (shown in Figure 5 ), with a higher frequency component imposed on it (in Figure 4(c)). Camera rotation, even with these small amplitudes, can have measurable influences, as shown in Figures 6(a) and 6(b), in which the horizontal and vertical displacements are displayed for a series of consecutive images. Both the horizontal and vertical displacements are in the order of about 0.01 to 0.1 pixels for a $75 \mathrm{~mm}$ lens, thus being quite considerable. This is consistent with the generated images, which have the results displayed in the same figures for the $75 \mathrm{~mm}$ system. The data from this experiment will be used in the simulated data in section 3 to expand from a 2D-DIC setup to a stereo-DIC setup.

\section{Simulation of camera motion}

\subsection{Introduction}

It is cumbersome to experimentally check the influence of camera rotation on a stereo-DIC measurement system due to the small order of magnitude of the rotations, the unknowns during an experiment, the combined effect of several influences, and the interaction of both cameras translating and rotating simultaneously. Therefore, the authors chose to use a stereo-DIC simulator (presented earlier in [2] and used in [7] for stereo-DIC uncertainty quantification), to fully capture the nature of the errors caused by camera rotation for a stereo-DIC setup. Because the images are numerically generated, the exact shape and deformation of the specimen in the region of interest (ROI) and rotation angles for both cameras are known and all error sources except camera motion can be ignored. The simulated stereo-DIC images are subdivided into two categories. The first category simulates the experiment described in 2.1, in which one camera is placed perpendicular and 


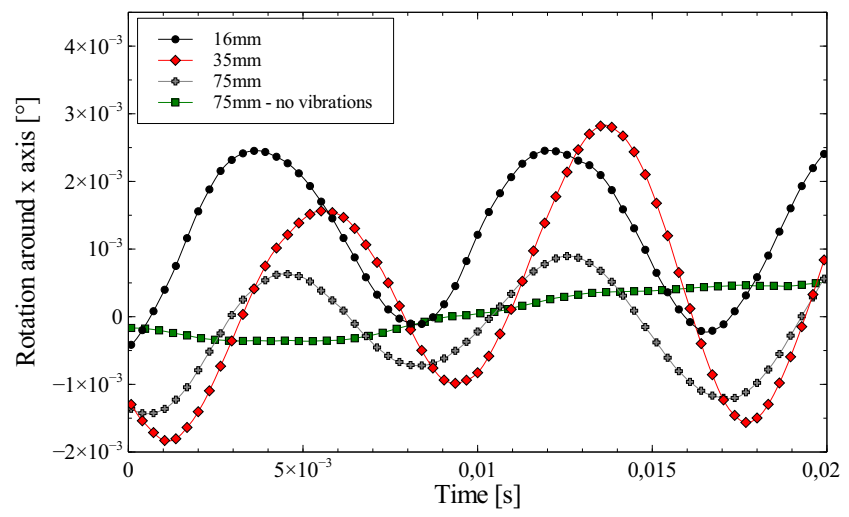

(a) Rotation around the $\mathrm{X}$-axis

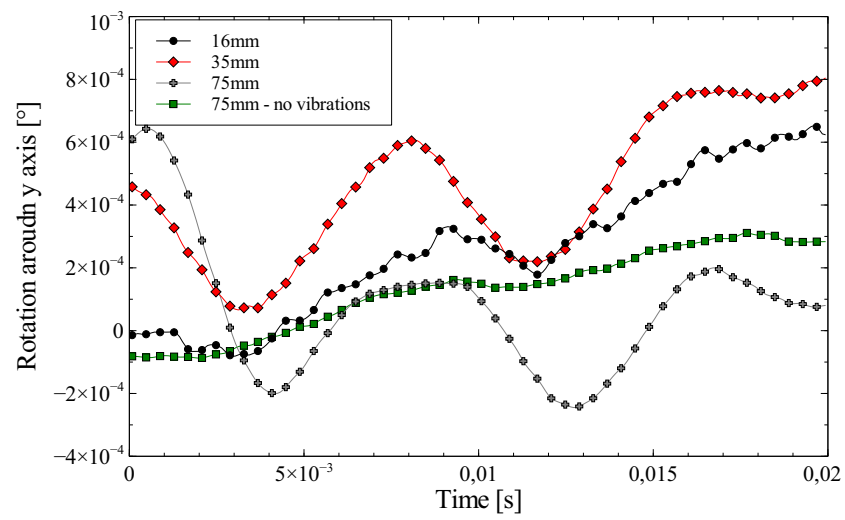

(b) Rotation around the Y-axis

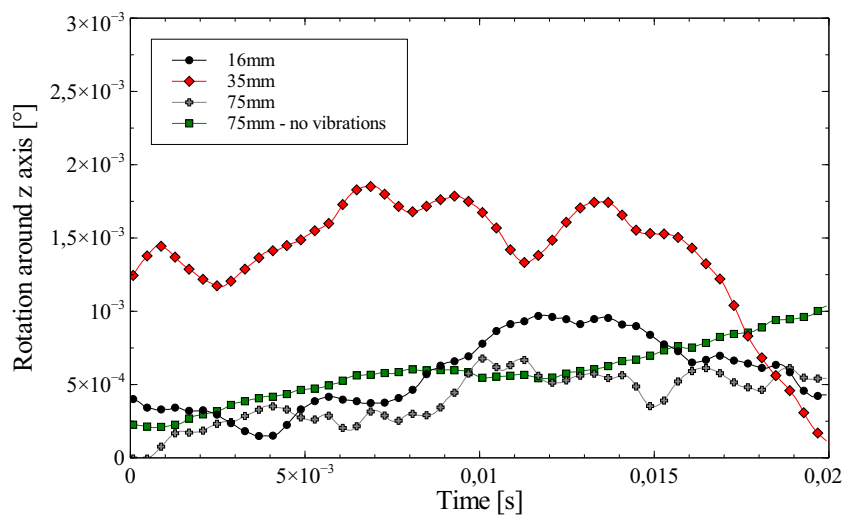

(c) Rotation around the Z-axis

Fig. 4: Camera rotations in simulated industrial environment experiment 


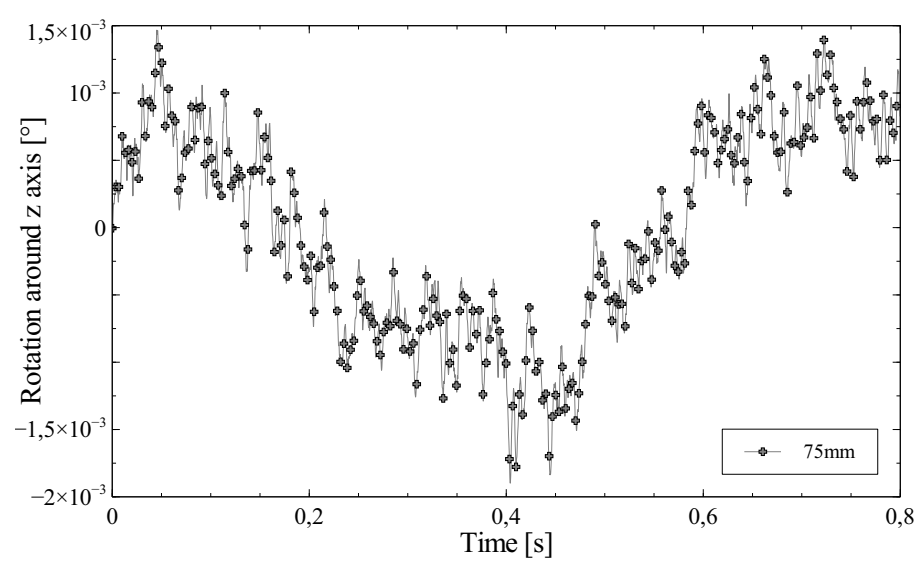

Fig. 5: Rotation around the horizontal out-of-plane camera axis
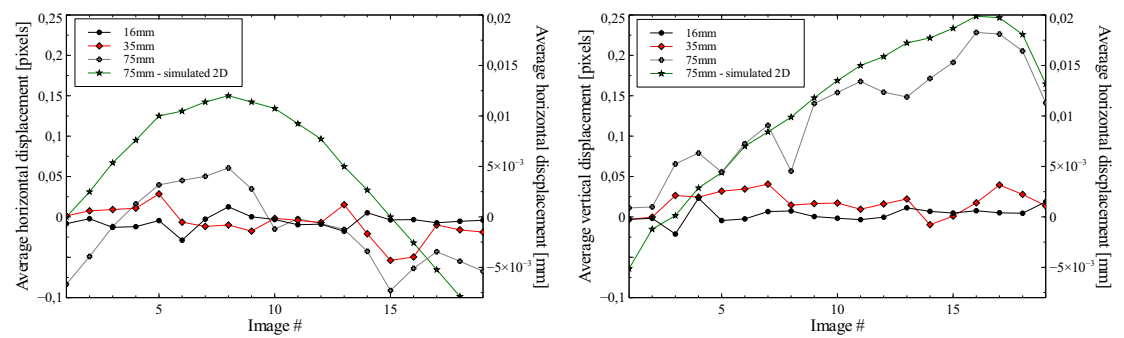

(a) Average horizontal displacement data (b) Average vertical displacement data from $2 \mathrm{D}-\mathrm{DIC}$ from $2 \mathrm{D}$-DIC

Fig. 6: Average displacements for a 2D-DIC system with camera rotations present

one is angled. Simulations in this category are used to both validate the simulations with the experimental data while simultaneously checking the influence of the focal length of the lenses being used and the stereo angle of the DIC rig. The second category of simulations focused on a bulging experiment and the influence of camera rotation on the obtained displacements and strains.

\subsection{Flat plate}

The influence of camera rotation on the measured shape, motion, and strain is first investigated on a flat plate with a simulated stereo-DIC setup in which one camera is placed perpendicularly for validating the experimentally obtained 2DDIC results (described in section 2.2), while also providing data concerning the impact of camera rotation on a stereo-DIC system. The simulated stereo setups 
Table 1: Evaluated configurations

\begin{tabular}{cccll}
\hline Setup nr & Angle cam 0 $\left[^{\circ}\right]$ & Angle cam 1 $\left[^{\circ}\right]$ & $\begin{array}{l}\text { Stereo } \\
\left.\text { angle }{ }^{\circ}\right]\end{array}$ & $\begin{array}{l}\text { Focal } \\
\text { length } \\
\text { lenses } \\
{[\mathbf{m m}]}\end{array}$ \\
\hline 1 & 0 & 10 & 10 & 16 \\
\hline 2 & 0 & 20 & 20 & 16 \\
\hline 3 & 0 & 10 & 10 & 35 \\
\hline 4 & 0 & 20 & 20 & 35 \\
\hline 5 & 0 & 10 & 10 & 75 \\
\hline 6 & 0 & 20 & 20 & 75 \\
\hline
\end{tabular}

are enumerated in Table 1, while the imposed camera rotations are all the possible combinations of rotations for both cameras (of all the 64 possible combinations, the worst case amplitude of the rotation around each axis can be seen in Table 2). By iterating over all possible combinations of rotations the worst combinations of camera rotations can be found. Both the experimental and the simulated images from the perpendicular camera were analyzed with the 2D-DIC settings given in Table 3 to validate the simulated images with the experimental results described in section 2. Similar results as in Figures 6(a) and 6(b) were obtained for all cases when analyzing the images from the perpendicular camera only, thus confirming the validity of the simulations.

Table 2: Imposed maximum camera rotations

\begin{tabular}{lll}
\hline $\mathbf{X}$ axis $\left[{ }^{\circ}\right]$ & $\mathbf{Y}$ axis $\left[^{\circ}\right]$ & $\mathbf{Z}$ axis $\left[{ }^{\circ}\right]$ \\
\hline $1.1 \cdot 10^{-3}$ & $5 \cdot 10^{-4}$ & $3 \cdot 10^{-4}$ \\
\hline
\end{tabular}

The stereo-DIC results are shown in Figures 7(a), 7(b) and 7(c) for the displacements and Figures $8(\mathrm{a}), 8(\mathrm{~b})$ and $8(\mathrm{c})$ depict the strain bias caused by camera rotation. The graphs represent the average displacement or strain over the entire ROI (region of interest) for a setup in which both cameras rotated away from each other around their respective vertical axis with an angle of $5 \cdot 10^{-4}$ degrees, while both cameras also rotated $1.1 \cdot 10^{-3}$ degrees down.

From the simulated images the following conclusions could be made concerning camera rotation during a stereo-DIC experiment:

- The focal length determines the magnitude of the error of the in-plane components, rather than the stereo-angle. The larger the focal length, the larger the errors induced by the camera rotation. This can be explained intuitively; due 


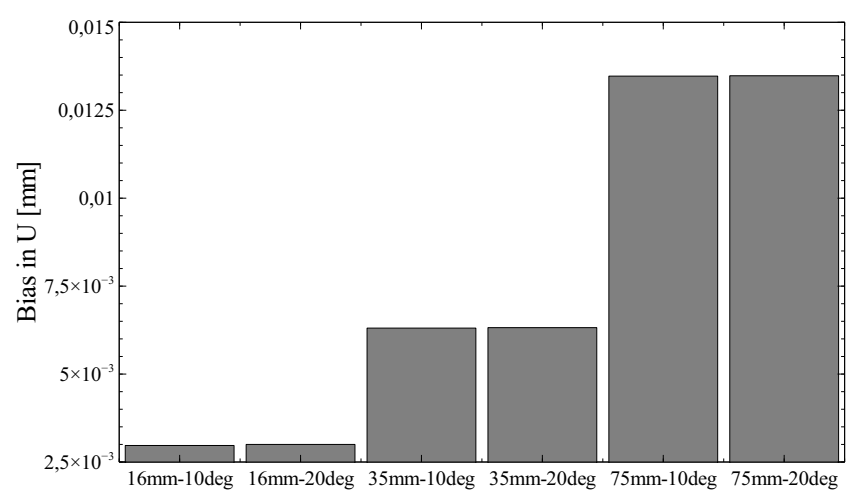

(a) Horizontal displacement bias due to camera rotation

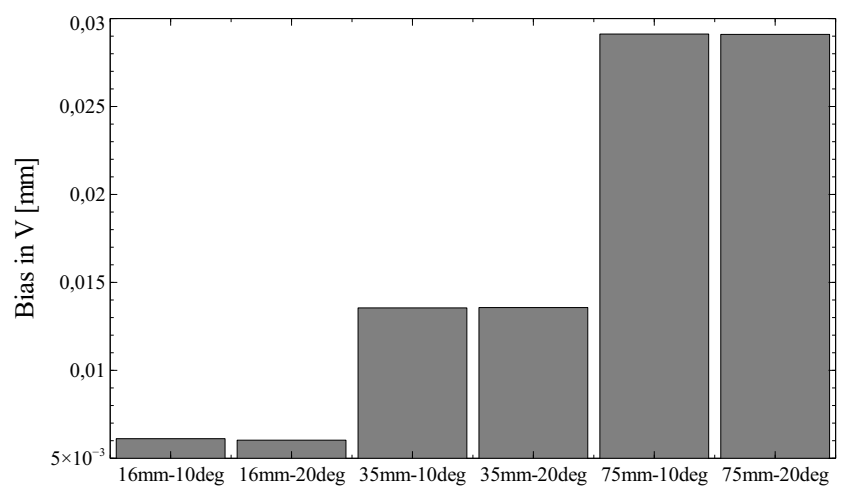

(b) Vertical displacement bias due to camera rotation

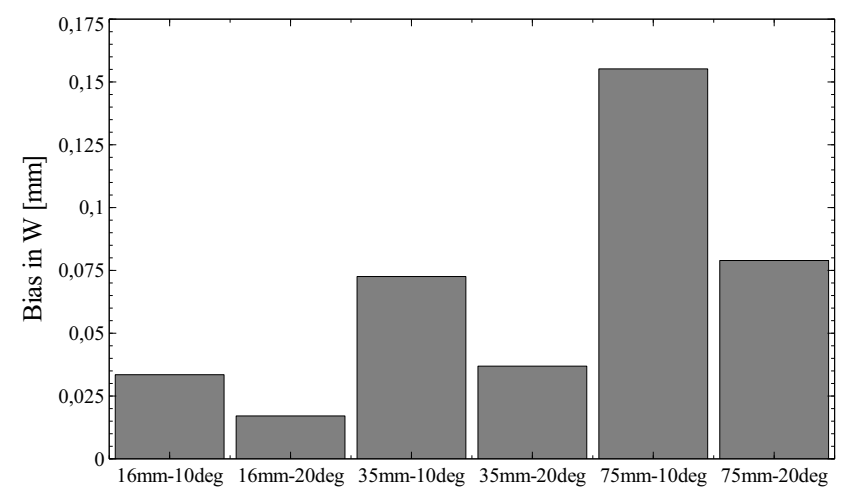

(c) Out-of-plane displacement bias due to camera rotation

Fig. 7: Mean displacement bias due to camera rotation 


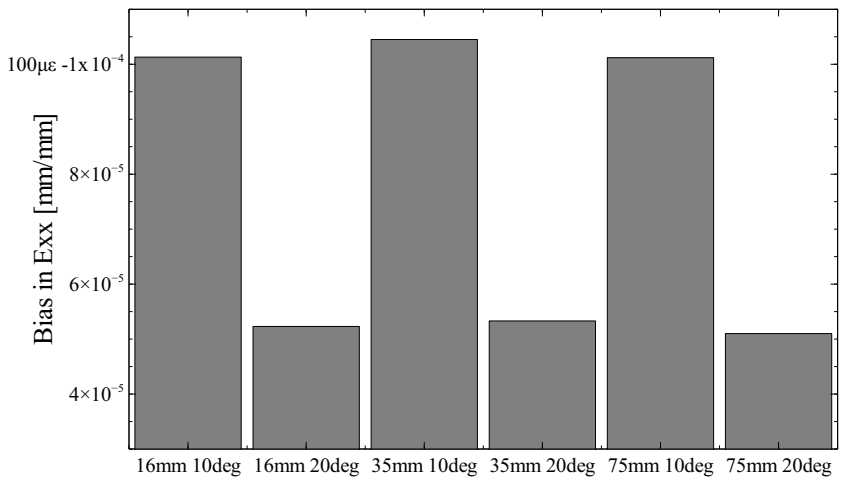

(a) Exx strain bias due to camera rotation

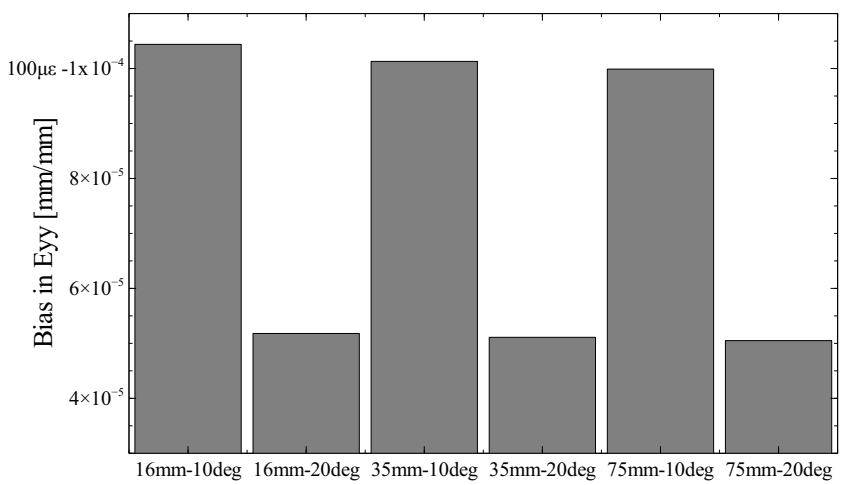

(b) Eyy strain bias due to camera rotation

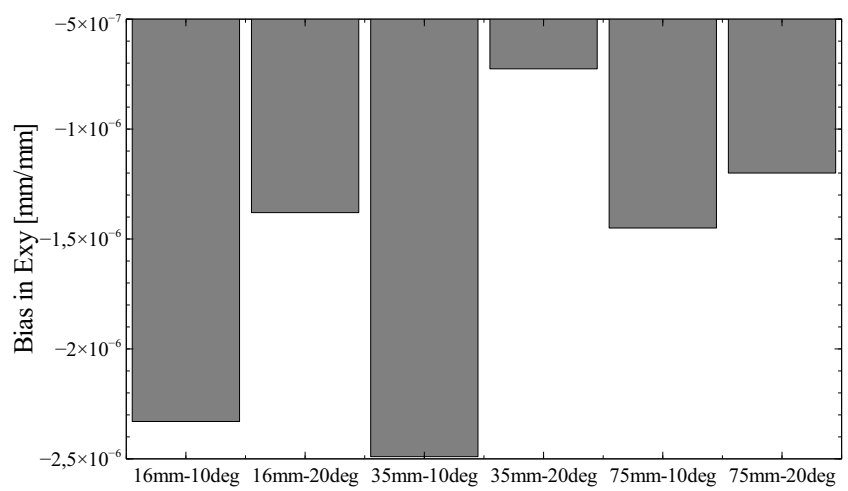

(c) Shear strain bias due to camera rotation

Fig. 8: Mean strain bias due to camera rotation 
Table 3: Adopted DIC-settings

\begin{tabular}{lll}
\hline Parameter & Value \\
\hline Camera resolution [pixels] & $1624 \times 1234$ \\
\hline Camera noise level [\% of dynamic range] & 0.5 & \\
\hline mm per pixel & 0.082 & \\
\hline Subset size [pixels] & 17 & \\
\hline Step [pixels] & 8 & Euler- \\
\hline Strain window [pixels] & 7 & \\
\hline VSG size [pixels] & 65 & \\
\hline Strain convention & Logarithmic & \\
& Almansi & \\
\hline Interpolation & B-spline & \\
\hline Transformation & Quadratic & \\
\hline
\end{tabular}

to the stand-off of the lens, the same camera motion will represent a higher motion at the target.

- The out-of-plane error is influenced by both the stereo-angle and the focal length: the errors are larger when the focal length is larger and when the stereo angle is smaller.

- Shear strains are barely influenced by the stereo angle of the setup, while the normal strains display higher errors when a lower stereo angle is present.

- The in-plane horizontal displacement $(\mathrm{U})$ is always biased when there is a rotation around the $\mathrm{y}$-axis, as indicated in Table 4.

- The in-plane vertical displacement (V) is biased when both cameras rotate around the $\mathrm{x}$-axis in the same direction, as shown in Table 4.

- The measured out-of-plane displacements (W) and the normal strains (Exx, Eyy) are mainly influenced when the cameras rotate opposite to one another around the y-axis. A visualization of this, together with the order of magnitude of the bias can be found in Table 4 .

- Shear strains (Exy) do not seem to be influenced by the direction of the camera rotation, even though the cameras move around all axes simultaneously.

Table 4: Camera motion combinations creating the largest errors

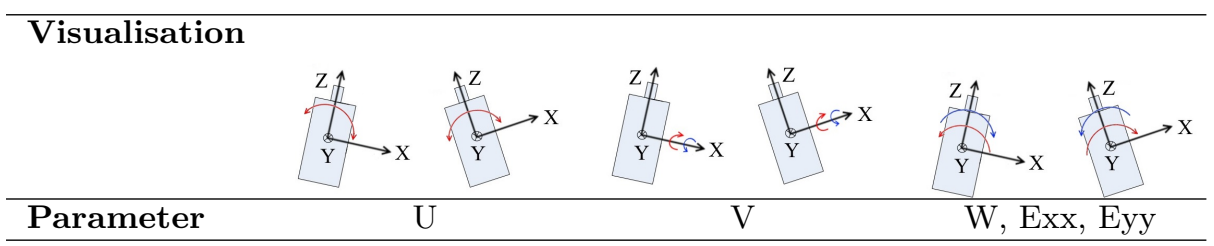


These conclusions will be illustrated further in the next section on a bulging plate simulation.

\subsection{Bulging experiment}

In this section, a bulge experiment is simulated to further investigate the observations made in section 3.2, but now with a deforming specimen. A round flat plate is clamped on the circumference and pressure is applied on the back side so that the plate bulges out and a heterogeneous deformation- and strain-field is obtained. The cameras are both angled at half of the stereo angle and are looking at the center of the (originally) flat plate. The details of the experiment can be seen in Table 5 and the adopted DIC-settings are the same as for the flat-plate simulation (see Table 3), which is appropriate because the same field of view (FOV) and speckle pattern were used for both simulations.

Table 5: Properties of bulge experiment

\begin{tabular}{ll}
\hline Parameter & Value \\
\hline Radius plate $[\mathrm{mm}]$ & 50 \\
\hline Thickness plate $[\mathrm{mm}]$ & 3 \\
\hline Young's Modulus material $[\mathrm{GPa}]$ & 210 \\
\hline Poisson ratio material & 0.30 \\
\hline Pressure [Bar] & 119 \\
\hline Stereo angle DIC system $[\mathrm{deg}]$ & 30 \\
\hline Focal length lenses $[\mathrm{mm}]$ & 30 \\
\hline Lens distortion factor $\kappa 1\left[\mathrm{pixels}^{-2}\right]$ camera $1^{-2}$ & 0.66 \\
\hline Lens distortion factor $\kappa 1\left[\right.$ pixels $^{-2}$ camera 2 & 0.25 \\
\hline
\end{tabular}

The simulated images are analyzed with MatchID-stereo with perfect calibration parameters (as exported by the simulator), thus ignoring the influence of poor calibration data. The reference images are the images of the flat plate with a stationary setup and these are correlated with the images that represent the deformed state of the plate and with a rotating setup and varying rotation angles of the cameras. An extraction through the vertical axis of the plate (indicated as a green dashed line in Figure 9(a)) is performed for a stationary setup and for setups in which both cameras rotate away from one another around their respective vertical axis with an angle of $0.001^{\circ}, 0.005^{\circ}, 0.01^{\circ}$ and $0.015^{\circ}$. The full field results for a stationary setup can be seen in Figures 9 (a) to 9 (c), while the extraction results can be seen in Figures 10 and 11. It is again clear that displacements are 


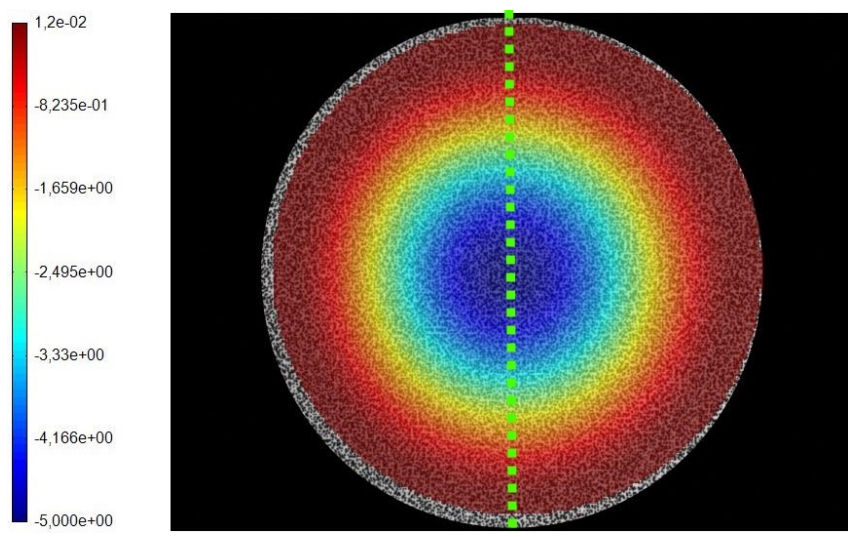

(a) Out-of-plane displacement [mm]

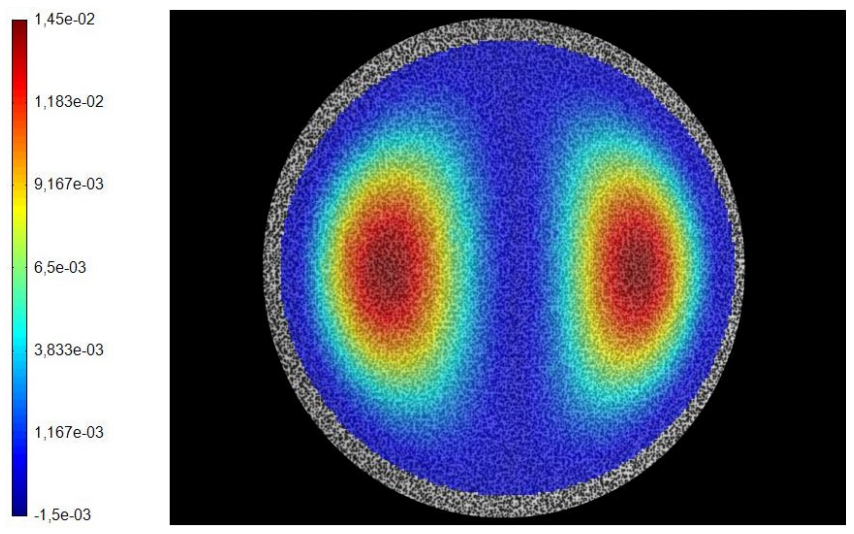

(b) $\operatorname{Exx}[\mathrm{mm} / \mathrm{mm}]$

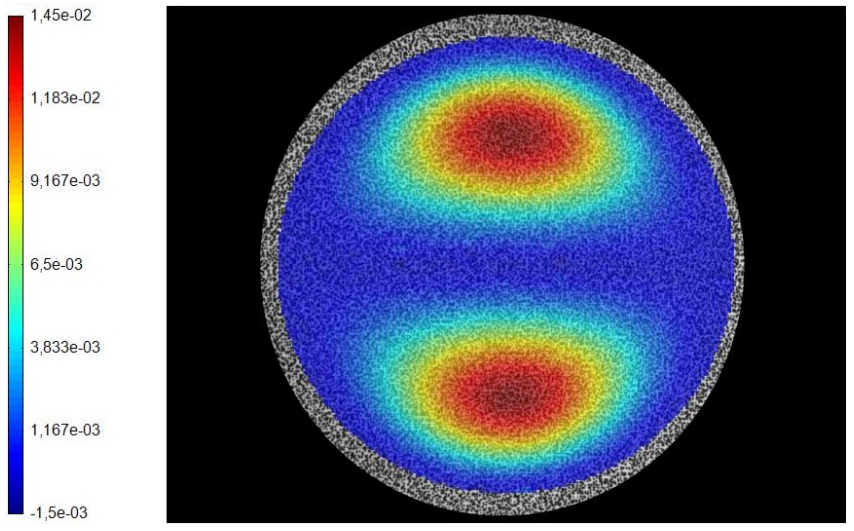

(c) Eyy $[\mathrm{mm} / \mathrm{mm}]$

Fig. 9: Bulge simulation - displacements and strains when no camera rotation is present 


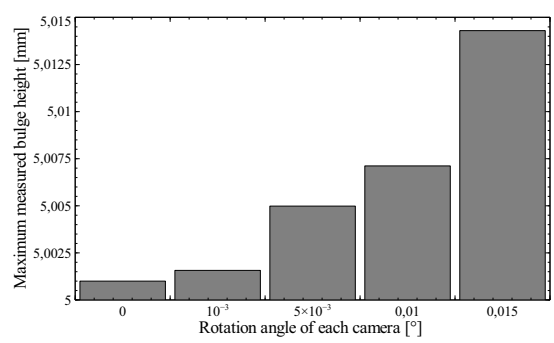

(a) Influence on measured bulge height

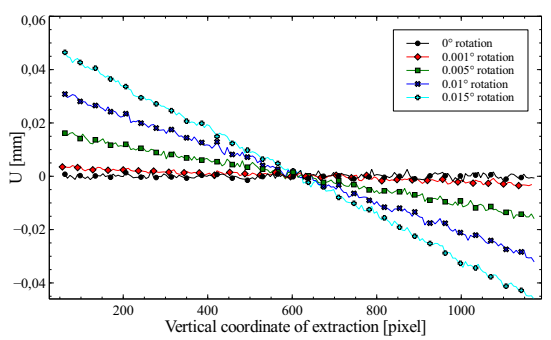

(b) Vertical in-plane displacement

Fig. 10: Bulge simulation - influence of rotation angle on displacements

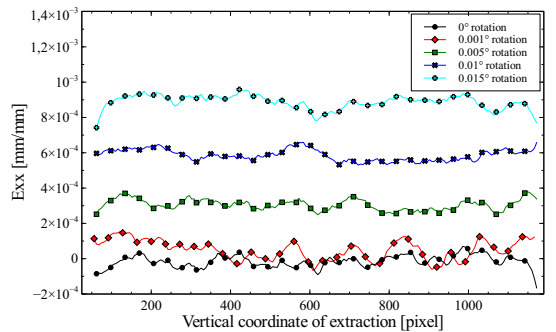

(a) $\operatorname{Exx}$

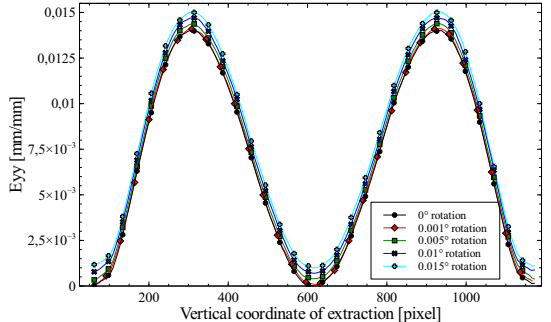

(b) Eyy

Fig. 11: Bulge simulation - influence of rotation angle on strains

biased in a linear manner, in which the slope is dictated by the angle of rotation, while strains display an offset that is proportional to the rotation angle.

\section{Detection and correction of camera rotation}

The effect of camera rotation is often present during DIC experiments, especially in the case of higher focal length lenses and lower stereo angles as shown in section 3. To improve measurements it is thus advisable to modify the setup to minimize the effects as much as possible. This is, however, not always possible. In the case of blast experiments for example, it is not always feasible to remove environmental vibrations and longer focal length lenses and longer stand-off distances are often required to protect the equipment. In those cases it is recommended to do a postprocessing stage to remove the effects of camera rotation (e.g. [11]). In section 4.1 we propose a camera rotation detection method and we investigate two methods that can be used for removing camera rotation. The first method was temporal averaging in section 4.2. The second proposed method for removing camera rotation is introduced in section 4.3 uses numerical rectification of the acquired 
images, based on the earlier introduced simulator and tracking stationary fiducials in the background. This method was already proven valuable for correcting nonperpendicular camera placement in 2D-DIC [?], however in a somewhat modified form (only one registration of the non-perpendicularity of the camera instead of continuous correction of the camera rotation). The authors refer to the literature (e.g., [12]) for a third possible manner of counteracting camera motion: camera self-calibration. This method is however not investigated here.

\subsection{Detection of camera rotation}

Because camera rotation can introduce large errors, it is of utmost importance to check whether rotation is present, and at what magnitude. The easiest way of doing this is by taking static images before testing and checking whether motions are present in the displacement field. By performing this check, environmental vibrations can be detected pre-test. However, vibrations can be caused by the test itself, e.g., during blast testing (e.g., [11]). These vibrations cannot be detected in advance and the user must be aware that these vibrations can be present. One possible approach for detecting these vibrations is by analyzing the test images in two ways; once for the region of interest (i.e., the specimen being investigated) and once using a stationary background scenery. The background scenery should yield a stationary displacement field, however, if motions are present in the analysis of the background it is clear that the cameras moved and that a correction is needed. To assist in this process, we thus focus on a metric that some vendors offer in their DIC packages (e.g., MatchID [13]): the epipolar distance. When a triangulation is performed the search for the corresponding points between both cameras is optimized by using the epipolar constraint, limiting the possible corresponding location of a point in the second camera to a line in the FOV by using the calibration data and the location of the point in the first camera [14]. However, since the calibration data is never perfect, a small deviation from the epipolar line is always present and the epipolar distance plot can thus be used to check both the initial calibration quality as well as any change of calibration quality, i.e., in this case the change in the relative orientation of the cameras by environmental vibrations. The maximum epipolar distance in the images of the initial flat plate from the bulging simulation (see section 3.3) are plotted versus the rotation angle of the cameras. Please note that the original, good calibration parameters (those with the known parameters and no camera rotation) are used for analyzing all images with $30 \mathrm{~mm}$ lenses as defined in Table 5 and for a modified setup in which $200 \mathrm{~mm}$ lenses are used. From Figure 12 we can clearly note that the reported epipolar distance is increasing proportionally with the camera rotation angle, thus 
making it a valid metric for detecting camera rotation. However, please note that no change in epipolar distance is detected if the cameras move along the epipolar line, therefore care must be taken when using the epipolar line as the only error indicator.

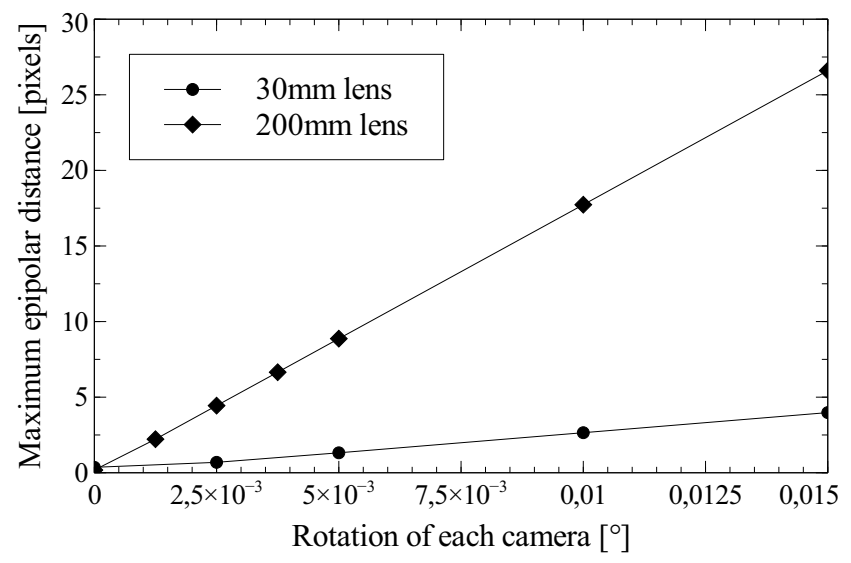

Fig. 12: Using the epipolar distance for detecting camera rotation

\subsection{Temporal averaging as correction tool}

Temporal averaging is the first correction method investigated for correcting the influence of camera rotation. In this case multiple images are taken at the same load step and these are then averaged over time in order to reduce the motion effect. This assumes that the calibration data represents the mean location of the cameras and that camera motion averages out over a longer period. To verify this technique, ten images were generated for setup number five in Table 1 (the flat plate setup, i.e. no motion should be measured), in which the cameras rotated sinusoidally around the $\mathrm{x}$ - and $\mathrm{y}$-axis with the amplitudes presented in Table 2 . The rotations were in phase and the ten images represent one period of rotation. The validity of temporal averaging is checked by varying the used number of images for averaging from two to ten by steps of two and the result for the measured out-of-plane motion is depicted in Figure 13.

It is clear from Figure 13 that temporal averaging does not always yield good results when attempting to remove camera rotation. But if the entire period of motion can be averaged, the results are returned to the noise-level of a stationary system. Temporal averaging for eliminating camera motion is thus only a valid 


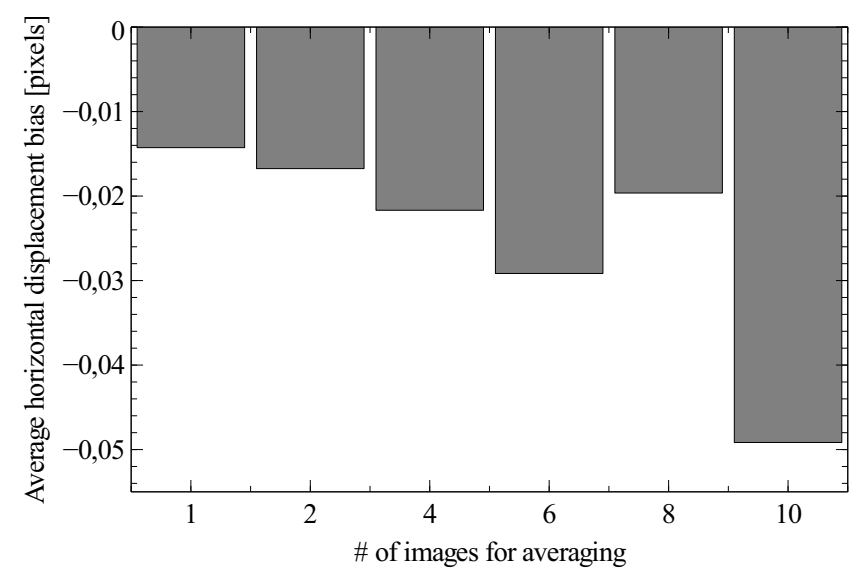

Fig. 13: Influence of temporal averaging on out-of-plane motion.

correcting method when the frequency of the camera rotation is known and when enough images are averaged to encompass a multitude of the period.

\subsection{Image rectification as correction tool}

The second proposed technique involves the use of the simulator (in a slightly adapted form) and the tracking of background regions to numerically rectify the images from each load step and each camera view. The basic assumption behind this technique is that the background in the FOV (e.g., mountains in an outdoor experiment or a wall in an indoor experiment) does not move during the experiment, yielding reference points from which camera motion can be estimated and corrected. Indeed, by tracking one region at each corner of the FOV the transformation of the image due to camera motion can be identified and then corrected for by using the simulator. The four tracking regions are monitored for each camera by using a standard 2D-DIC algorithm, taking the lens distortions for the respective cameras into account. Each tracking region has the size of at least one subset, of which the minimal size is determined based on the criterium of having three speckles per subset (the authors refer to [15] for more information on subset size selection). However larger regions of interest are recommended to obtain the best accuracy for the estimated motion. Each image is then corrected, based on the apparent motions of the tracked regions by numerically deforming the image by imposing an opposite transformation. Numerical rectification was previously also succesfully performed for correcting out-of-plane compensation in 2D-DIC experiments due to non-perpendicular camera setup in [?]. Rectified images are obtained for each camera and each load step and are then used for performing 
stereo-DIC triangulation. The main advantage of this technique is that all motions are corrected at once and that possible drift of the external calibration data will not introduce extra errors since these are corrected for. The downside of this technique is that the four tracking regions must remain in the FOV at all times and that the results are dependent on the quality of tracking of these regions and their remaining stationairy. If the lens distortions are poorly calibrated and the reference regions are small and on the edge of the FOV (where the distortions are usually the highest due to the mainly radial nature of lens distortions) poor tracking data may result, thus deteriorating the quality of the correction. To minimize the errors, it is advisable to have tracking regions that encompass multiple subsets and thus using the average displacement of all subsets within one tracking region. Some small errors are also possible due to the numerical rectification of the images, which will be compromised when the speckles show indications of aliasing, increasing interpolant errors. We would also like to refer to a paper by Pan et. al [16], which describes a similar method for correcting errors in 2D-DIC induced by camera motion or specimen out-of-plane motion. Here a reference sample is rigidly attached to the specimen and the corrupted 2D-DIC data is compensated afterwards by using a parametric model. The authors were however unable to test this method for stereo-DIC since it would require changes in the source code of a stereoDIC package to impose this compensation method also during the cross-correlation step. A comparison between both methods is however given in [?] for 2D-DIC, indicating that both methods have equal compensation capabilities. The validity of this compensation technique is evaluated on the experimentally obtained images from section 2.1 (more specifically for the $75 \mathrm{~mm}$ lens), which showed a drift in the vertical displacement of a longer period of time. Both the original, measured displacements, as well as the compensated ones, can be seen in Figure 14, in which the in-plane components show remarkable improvement after compensation (the standard deviation of the compensated images was below $10^{-3}$ pixels for both the horizontal and vertical displacement).

\section{Conclusion}

This paper describes the influence of camera motion in stereo-DIC by generating images in a stereo-DIC simulator and by imposing changing camera orientations. Camera motion was measured by mimicking an industrial environment by placing a compressor near a 2D-DIC setup and the motion of both the camera body and the lens was measured. Section 2 demonstrated the result of these camera rotations on DIC and indicated that, even though the order of magnitude of the rotations is very small (in the order of magnitude of $0.001^{\circ}$ to $0.0001^{\circ}$ ), camera rotation 


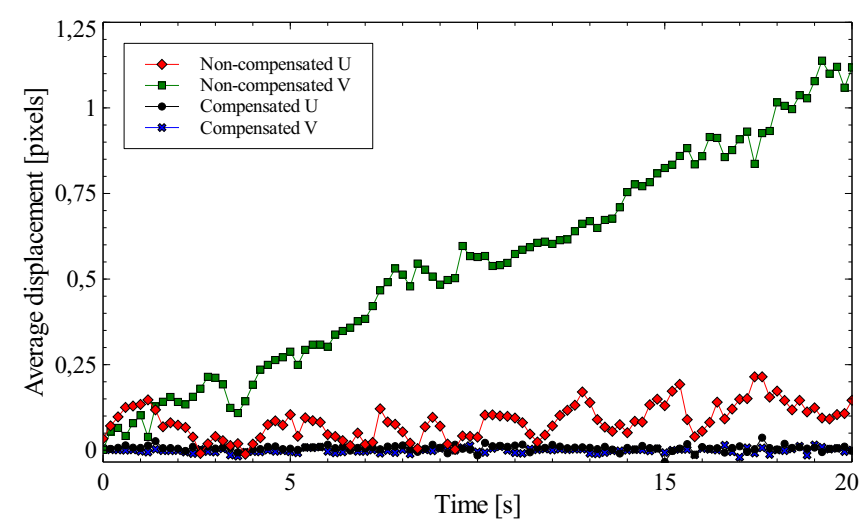

Fig. 14: Numerical rectification of images for camera rotation compensation $2 \mathrm{D}-\mathrm{DIC}$ results

cannot be neglected and should be considered when environmental vibrations are present. Section 3 expanded the 2D-DIC findings to stereo-DIC using simulated images. The 2D-DIC results of section 2 were first validated between the simulation and the experiment to verify the simulation approach. The stereo-DIC results indicated that the influence of camera rotation is larger when lower stereo-angles and higher focal length lenses are used in a stereo-DIC setup. It is also clear that displacements show a linear behavior, while strains are biased by a constant factor.

A valid camera motion correction method, based on numerically rectifying images, was shown in section 4.3. Changing camera orientations are identified by tracking the background with 2D-DIC and an inverse mapping function can retrieve the image that would have been taken with a static camera, thus eliminating the errors induced by camera motion.

It is clear that camera rotation should not be neglected, especially during experiments in which high environmental vibrations are present (e.g., industrial environments, blast loading experiments, etc.) and compensation methods may need to be applied in those cases to improve the quality of stereo-DIC measurements.

\section{Acknowledgements}

The authors would like to thank Sandia National Labs for performing the motion measurements described in section 2. Sandia National Laboratories is a multimission laboratory managed and operated by National Technology and Engineering Solutions of Sandia LLC, a wholly owned subsidiary of Honeywell In- 
ternational Inc., for the U.S. Department of Energy's National Nuclear Security Administration under contract DE-NA0003525.

\section{References}

1. Helffrick M. N., C. Niezrecki, Avitabile P., and Schmidt T. 3d digital image correlation methods for full-field vibration measurement. Mechanical Systems and Signal Processing, 25(3):917-927, April 2011.

2. Balcaen R., Wittevrongel L., Reu P.L., Lava P., and Debruyne D. Stereo-dic calibration and speckle image generator based on fe formulations. Experimental Mechanics, 57(5):703$718,2017$.

3. Sutton M. A., Orteu J.J., and Schreier H. W. Image correlation for shape, motion and deformation measurements. Springer Science, 2009.

4. Bornert M., Brmand F., Doumalin P., Dupr J.-C., Fazzini M., Grdiac M., Hild F., Mistou S., Molimard J., Orteu J.-J., Robert L., Surrel Y., Vacher P., and Wattrisse B. Assessment of digital image correlation measurement errors: methodology and results. Experimetal Mechanics, 49(3):353-370, June 2009.

5. Wang Y.Q., Sutton M.A., Ke X. D., W. Schreier H., L. Reu P., and J. Miller T. On error assessment in stereo-based deformation measurements-part 1. Experimental mechanics, 51(4):405-422, April 2011.

6. Ke X. D., W. Schreier H., A. Sutton M., and Wang Y. Q. On error assessment in stereobased deformation measurements-part 2. Experimental mechanics, 51(4):423-441, April 2011.

7. Balcaen R., Reu P.L., Lava P., and Debruyne D. Stereo-dic uncertainty quantification based on simulated images. Experimental Mechanics, 2017.

8. B Pan, W. Shi, and G Lubineau. Effect of camera temperature variations on stereo-digital image correlation measurements. Applied Optics, 54(34), 2015.

9. Bing Pan, Dafang Wu, and Liping Yu. Optimization of a three-dimensional digital image correlation system for deformation measurements in extreme environments. Applied Optics, 2012.

10. M A Sutton, J.H. Yan, V. Tiwari, Hubert Schreier, and J. J. Orteu. The effect of out-ofplane motion on $2 \mathrm{~d}$ and $3 \mathrm{~d}$ digital image correlation measurements. Optics and Lasers in Engineering, 46:746-757, 2008.

11. Miller T. J., Schreier H. W., and Reu P. L. High-speed dic data analysis from a shaking camera system. In SEM annual conference, 2007.

12. Dang T., Hoffman C., and Stiller C. Continuous stereo self-calibration by camera parameter tracking. IEEE Transactions on Image Processing, 18(7):1536-1550, July 2009.

13. http://www.matchidmbc.com/.

14. Hartley RI and Zisserman A. Multiple view geometry in computer vision. Cambridge University press, 2004.

15. B. Pan, H. Xie, Z. Wang, K. Qian, and Z. Wang. Study on subset size selection in digital image correlation for speckle patterns. Optics Express, 16(10):7037-7048, 2008.

16. Bing Pan, Liping Yu, and Dafang Wu. High-accuracy 2d digital image correlation measurements using low-cost imaging lenses: implementation of a generalized compensation method. Measurement Science and Technology, 25, 2014. 